\title{
Stapling Through a Bougie During Sleeve Gastrectomy in a Superobese Patient-a Video Vignette
}

\author{
Christoph Bichler $^{1}$ - Julia Jedamzik ${ }^{1}$. Daniel M. Felsenreich ${ }^{1} \cdot$ Felix B. Langer $^{1} \cdot$ Magdalena Eilenberg $^{1} \cdot$ Natalie Vock $^{1}$. \\ Katharina Steinlechner ${ }^{1}$ • Jakob Eichelter ${ }^{1}$. Lisa Gensthaler ${ }^{1} \cdot$ Gerhard Prager $^{1}$
}

Received: 4 April 2020 / Revised: 9 June 2020 / Accepted: 15 June 2020 / Published online: 2 July 2020

(C) The Author(s) 2020

\begin{abstract}
Purpose Bariatric-metabolic surgery in superobese patients (BMI $\left.>50 \mathrm{~kg} / \mathrm{m}^{2}\right)$ is very challenging indeed with little room for error. In many cases, a two-step procedure is required, since more complex primary bariatric procedures can be technically demanding and bearing a relevant risk for the patient. At our institution, laparoscopic sleeve gastrectomy (SG) is the preferred primary procedure, followed by a conversion to either SADI-S or Roux-en-Y gastric bypass (RYGB) after initial weight loss is achieved [1, 2]. This video aims at demonstrating the conversion from primary SG to RYGB due to an adverse event in a 45-year-old superobese female patient (weight, $170 \mathrm{~kg}$; BMI, $73 \mathrm{~kg} / \mathrm{m}^{2}$ ).

Methods An intraoperative laparoscopic video has been anonymized and edited to demonstrate the course of the operation on the patient mentioned above.

Results The start of the procedure was uneventful. After a successful mobilization of the greater curvature, the stomach was resected with an electronic stapling device guided by a firm 36-french bougie (Rüsch, Germany) towards the angle of His. Due to a limited view, a stapler was placed over the bougie, which resulted in the stomach being subtotally transected, the staples attaching the bougie to the sleeve about $5 \mathrm{~cm}$ from the gastroesophageal junction. Salvage surgery after removing the remnants of the bougie was a conversion to RYGB.

Conclusion When performing a bariatric-metabolic surgery in superobese patients, an extended skill level is required to provide a solution, should anything go wrong. Therefore, we suggest bariatric-metabolic surgery in superobese patients to be performed solely and specifically at high-volume centres.
\end{abstract}

Keywords Sleeve gastrectomy $\cdot$ RYGB $\cdot$ Video vignette $\cdot$ Bougie

\section{Introduction}

Bariatric-metabolic surgery in superobese patients (BMI > $\left.50 \mathrm{~kg} / \mathrm{m}^{2}\right)$ is very challenging indeed with little room for error. In many cases, a two-step procedure is required, since more complex primary bariatric procedures can be technically

Electronic supplementary material The online version of this article (https://doi.org/10.1007/s11695-020-04790-z) contains supplementary material, which is available to authorized users.

Gerhard Prager

gerhard.prager@meduniwien.ac.at

1 Division of General Surgery, Department of Surgery, Vienna Medical University, Waehringer Guertel 18-20,

1090 Vienna, Austria demanding and bearing a relevant risk for the patient. At our institution, laparoscopic sleeve gastrectomy (SG) is the preferred primary procedure, followed by a conversion to either SADI-S or Roux-en-Y gastric bypass (RYGB) after initial weight loss is achieved $[1,2]$.

\section{Purpose}

This video aims at demonstrating the conversion from primary SG to RYGB due to an adverse event in a 45 -year-old superobese female patient (weight, $170 \mathrm{~kg}$; BMI $73 \mathrm{~kg} / \mathrm{m}^{2}$ ). In preparation, the patient received a dietary counselling and was able to lose $9 \mathrm{~kg}$ (EWL (excess weight loss), 8\%; TWL (total weight loss), $5 \%$ ) on a low-carbohydrate diet. 


\section{Methods}

An intraoperative laparoscopic video has been anonymized and edited to demonstrate the course of the operation on the patient mentioned above.

\section{Results}

The start of the procedure was uneventful. After successful mobilization of the greater curvature, the stomach was resected with an electronic stapling device guided by a firm 36-french bougie (Rüsch, Germany) towards the angle of His. Due to a limited view, a stapler was placed over the bougie, which resulted in the stomach being subtotally transected, the staples attaching the bougie to the sleeve about $5 \mathrm{~cm}$ from the gastroesophageal junction. Salvage surgery after removing the remnants of the bougie was a conversion to RYGB. After surgery, the patient underwent an uneventful postoperative course.

\section{Discussion}

The decision to change strategies intraoperatively was made for the following reasons. Suturing the defect was eliminated due to the risks of postoperative stenosis and early leaks. Thus, the decision was made to staple proximal to the area harmed. A conversion to RYGB was chosen based on the fact that RYGB is a low-pressure system (as opposed to $\mathrm{SG}$ ) and is thus well suited to treat intra- and postoperative complications [3, 4]. Every bariatric patient should be informed preoperatively about a possible intraoperative conversion/change of strategy.

\section{Conclusion}

When performing bariatric-metabolic surgery in superobese patients, an extended skill level is required to provide a solution, should anything go wrong. Therefore, we suggest bariatric-metabolic surgery in superobese patients to be performed solely and specifically at high-volume centres.

Funding Information Open access funding provided by Medical University of Vienna.

\section{Compliance with Ethical Standards}

Conflict of Interest Christoph Bichler, Julia Jedamzik, Daniel M Felsenreich, Felix B Langer, Magdalena Eilenberg, Natalie Vock, Katharina Steinlechner, Jakob Eichelter, Lisa Gensthaler, and Gerhard Prager have no conflicts of interest or financial ties to disclose.

Human and Animal Rights The performed operation and its intraoperative change of strategy was in accordance with the ethical standards of the research committee of the Vienna Medical University and with the 1964 Helsinki declaration and its later amendments or comparable ethical standards. This article does not contain any studies with animals performed by any of the authors.

Informed Consent Written, informed consent was obtained from the individual participant included in this video.

Open Access This article is licensed under a Creative Commons Attribution 4.0 International License, which permits use, sharing, adaptation, distribution and reproduction in any medium or format, as long as you give appropriate credit to the original author(s) and the source, provide a link to the Creative Commons licence, and indicate if changes were made. The images or other third party material in this article are included in the article's Creative Commons licence, unless indicated otherwise in a credit line to the material. If material is not included in the article's Creative Commons licence and your intended use is not permitted by statutory regulation or exceeds the permitted use, you will need to obtain permission directly from the copyright holder. To view a copy of this licence, visit http://creativecommons.org/licenses/by/4.0/.

\section{References}

1. Iannelli A, Debs T, Martini F, et al. Laparoscopic conversion of sleeve gastrectomy to Roux-en-Y gastric bypass: indications and preliminary results. Surg Obes Relat Dis. 2016;12(8):1533-8.

2. Balibrea JM, Vilallonga R, Hidalgo M, et al. Mid-term results and responsiveness predictors after two-step single-anastomosis duodeno-Ileal bypass with sleeve gastrectomy. Obes Surg. 2017;27(5):1302-8.

3. Bellorin O, Lieb J, Szomstein S, et al. Laparoscopic conversion of sleeve gastrectomy to Roux-en-Y gastric bypass for acute gastric outlet obstruction after laparoscopic sleeve gastrectomy for morbid obesity. Surg Obes Relat Dis. 2010;6(5):566-8.

4. Saglam K, Aktas A, Gundogan E, et al. Management of acute sleeve gastrectomy leaks by conversion to Roux-en-Y gastric bypass: a small case series. Obes Surg. 2017;27(11):3061-3.

Publisher's Note Springer Nature remains neutral with regard to jurisdictional claims in published maps and institutional affiliations. 